MODELING, IDENTIFICATION AND CONTROL, 1991, VOL. 12, NO. 4, 179-205

doi:10.4173/mic.1991.4.2

\title{
On unsteady reacting flow in a channel with a cavity
}

\author{
IVAR ØYVIND SAND $\dagger$
}

Keywords: Unsteady reacting flow, vortex method, simple line interface calculation, conformal mapping.

The problem investigated is the stability of a flame anchored by recirculation within a channel with a cavity, acting as a two-dimensional approximation to a gas turbine combustion chamber. This is related to experiments of Vaneveld, Hom and Oppenheim (1982). The hypothesis studied is that hydrodynamic oscillations within the cavity can lead to flashback.

The method used is a semi-analytical-numerical technique where the conservation equations for enthalpy and fuel fraction are represented by the low-Machnumber combustion model of Ghoniem, Chorin and Oppenheim (1982). Burnt and unburnt gas are treated as incompressible fluids where the reaction zone acts as a source for volume expansion. The flame is modeled by a Lagrangian technique using a simple line interface calculation algorithm.

The turbulent flow field is determined using conformal mapping theory and the hybrid random vortex method. The vorticity generation takes place at the walls to achieve no slip, and is influenced by boundary-layer separation. To avoid locating the separation points $a$ priori the numerical viscous sublayer is extended continuously past the corners, and their singularities are in effect cut off by using locally a corner rounding technique within the conformal mapping.

The computed unsteady boundary-layer separation and reattachment of the non-reacting flow field agrees with unsteady boundary-layer theory. On the basis of the numerical simulations of the flame stability problem it is concluded that hydrodynamic oscillations within the cavity, combined with unsteady boundarylayer separation and reattachment can cause a flashback.

\section{Introduction}

One of the possibilities for reduction of oxides of nitrogen and particulate emission from gas turbine engines is the use of lean premixed prevaporized combustion, see Ganji and Sawyer (1980). The lower emission levels of oxides of nitrogen are caused by the lower peak combustion temperature compared to that of a conventional combustor which works on the basis of diffusion flames. The reduction of particulates, carbon monoxide and hydrocarbons is a consequence of more uniform combustion and an excess of air. The introduction of lean premixed prevaporized combustion to gas turbine combustors is however troubled by flame stability problems, such as blowout and flashback. In this work we focus on the latter. A general review of reasons for flashback is given in Plee and Mellor (1978).

Keller et al. (1981) studied experimentally the stability of a flame anchored behind a backward-facing step in lean premixed propane and air. They found three modes of

Received 12 December 1991.

†Chr. Michelson Institute, Department of Science and Technology, N-5036 Fantoft, Bergen, Norway.

Reprinted from J. Fluid Mechanics, Vol. 229, 1991 with permission from Cambridge University Press. 
unstable flame motion. One of these, which was metastable and made a characteristic humming sound, appeared as a response to acoustic excitation. A second mode, associated with a travelling wave pattern of interacting vortices, developed into the third mode associated with growth and convection of recirculation vortices, which in turn developed into flashback depending on the flow conditions. The dominating frequency periods of these modes of flame motion were lower than that of the humming, thus verifying their hydrodynamic nature. The experiments of Vaneveld, Hom and Oppenheim (1982), which also confirm these findings for a channel with a cavity geometry, show that hydrodynamic oscillations are the possible cause of many of the flashbacks reported within combustors. The motivation for this work is that increased knowledge about the hydrodynamic aspects of the combustion instabilities may help us in controlling them. Hence acoustic excitation of the flow field is excluded in this study. Based on experimental observations our flame stability problem is classified as taking place in the regime of reaction sheets where the chemical times are much shorter than the transport times, causeing the combustion to occur in thin sheets wrinkled by the turbulence. However, these sheets may be multiply connected depending on the turbulence intensity range, see Bray (1980). The reaction rate for converting unburnt fluid into burnt fluid is assumed to be proportional to the flame area, and the local burning speed relative to the flow field. By this simplification, the local burning speed becomes a function of fuel type, concentration of reactant and oxidizer, initial temperature, pressure, turbulence intensity, flame stretch and Lewis number.

However, since the combustion is premixed, the dependence of the local burning speed on fuel type and concentration of reactant and oxidizer is given once the mixture is chosen. Also the venting area is so large that the pressure varies little once the initial state variables are chosen. Theoretically the Landau instability, which is a local hydrodynamic instability based on the energy release across a thin flame, should increase flame wrinkles once they have been established. However, in lean premixed propane and air, where the effective Lewis number is greater than one, the local burning speed decreases for positive and increases for negative flame stretch respectively, and acts against unstable growth of flame wrinkles, see Law (1988). As a further simplification we therefore assume the local burning speed to be independent of flame stretch and reduced to that of a one-dimensional flame. Consequently the main effect of turbulence on the combustion rate is assumed to be enhancement of the flame area through wrinkling and folding. This may be a rough approximation in reality since it requires high space and time resolution to resolve numerically the geometry of the flame front and its motion.

In an unbounded region in a non-rotating reference frame, the only vorticity generation is caused by the baroclinic effect where unsteady pressure waves interact with the flame. See Ashurst and McMurty (1989) for work on modeling combustiongenerated vorticity in an unbounded flow field. However, when the flow field is bounded, the major source of vorticity is the shear force from the geometry acting on the fluid. The vorticity shearing, which increases the width of a mixing layer downstream of a boundary-layer separation, enhances the flame area drastically and thereby increases the overall combustion rate, provided the turbulence intensity is not too high or the mixture too lean which results in blowout of the flame as demonstrated by Ganji and Sawyer (1980). By prescribing the local burning velocity we have in principle neglected the possibility of flame extinction by turbulent straining of the flame. However, since we intend to study flashback and not blowout, this limitation should not affect the analysis significantly. 
The flow field is assumed to be two-dimensional. This excludes vortex stretching in the governing equations. However, the leading and the trailing edge of the cavity dominate the creation and destruction of vortices while the vortex stretching carried out by the smooth sidewalls, see the experiments of Vaneveld et al. (1982), takes place normal to the flow direction and is expected to be negligible.

There are several methods for solving the governing equations numerically, such as finite-difference, finite-element and vortex methods. The choice made here is motivated by the need to resolve numerically the unsteady boundary-layer separation and reattachment which characterizes cavity flow. A direct solution procedure by Ghoniem, Chorin and Oppenheim (1982) is used to solve an approximation to the governing equations for turbulent combustion in the low-Mach-number range neglecting acoustic waves. The flame front is represented as a constant-pressure deflagration wave superimposed on the turbulent flow field. The energy release is based on the assumption of conservation of enthalpy in a reference frame following the flame which is modeled by a Lagrangian technique using a simple line interface calculation algorithm. Huygen's principle is used to avoid computing the normal unit vector to the flame. This makes it possible to represent a multi-connected flame surface.

The exothermic process contributes to the velocity field via volume sources. The turbulent flow field is computed by the hybrid random vortex method which takes near-well regions into account. In this model the turbulent velocity fluctuations are induced by small and large groups of vortices affecting each point in space for a short or long time respectively as they pass by. To maintain no slip, vorticity is generated at the walls as vortex sheets which only induce velocity locally within a numerical viscous sublayer. Vortex sheets which leave this layer are redefined as vortex blobs which affect the velocity field globally, and back to vortex sheets if they re-enter the viscous sublayer. In their original work Ghoniem et al. (1982) used this model to simulate a flame stabilized behind a rearward-facing step. The flame motion was unsteady; however, the flame remained attached to the step edge.

The main problem investigated in this work is the stability of a flame anchored by the recirculation within a cavity. The hypothesis studied is that hydrodynamic oscillations of unsteady character can lead to flashback. To resolve the interaction of hydrodynamic oscillations within the cavity with unsteady boundary-layer separation and reattachment, the accuracy of the numerical scheme is improved. This is done by moving the vortices in the physical space by Simpsons fourth-order rule in time instead of using the original forward Euler scheme. To avoid locating the boundary-layer separation points a priori, the numerical viscous sublayer is extended continuously past the corners of the cavity and in effect the corner singularities are cut off by using locally a corner rounding technique within the conformal mapping, see $\S 7$.

The solution procedure is presented in $\$ 3-6$. Its accuracy and some of the conceptual ideas behind it are given in the Appendix. In $\S 9$ we discuss unsteady nonreacting and reacting flows and present our results.

\section{Mathematical formulation}

The following idealizations are made to simplify the problem.

(i) The fluid motion is two-dimensional.

(ii) The fluid consists of two homogeneous incompressible media, burnt and unburnt fluid. 
(iii) The flame can be represented as a constant-pressure deflagration wave acting as an interface between two incompressible media, and propagating with a speed equal to the local burning velocity relative to the fluid motion.

(iv) The exothermicity of combustion is manifested entirely by an increase in specific volume due to conversion of unburnt fluid into burnt fluid.

(v) Negligible buoyancy.

(vi) Turbulent mixing dominates over diffusion of species, enthalpy and mass fraction of burnt fluid.

(vii) The chemical reaction can be approximated sufficiently accurately by an irreversible one-step chemical reaction scheme.

(viii) The fluid satisfies the Newtonian constitutive assumptions for the relation between the stress tensor and the deformation tensor.

(ix) The boundaries are adiabatic.

On the basis of these assumptions the problem is determined by the following equations and conditions.

Conservation of mass gives

$$
1 / \rho D \rho / D t=-\nabla \cdot u .
$$

The Helmholtz vorticity equation in dimensionless form is

$$
D \omega / D t=1 / R e_{\mathrm{ch}} \nabla^{2} \omega .
$$

The equation of state for an ideal gas within the reaction zone, combined with the assumption of constant pressure across the reaction zone gives

$$
\rho_{\mathrm{u}} / \rho_{\mathrm{b}}=T_{\mathrm{b}} / T_{\mathrm{u}} .
$$

$T_{\mathrm{b}}$ is the adiabatic flame temperature at constant pressure for the chosen equivalence ratio of premixed reactant and oxidizer. $T_{\mathrm{u}}$ is the unburnt fluid temperature.

Assuming conservation of enthalpy in a reference frame following the flame combined with the definition of enthalpy for a complete reaction and constant-pressure deflagration we have

$$
h=\text { Constant }=\Delta h_{\mathrm{f}}+c_{p} \quad\left(T_{\mathrm{b}}-T_{\mathrm{ref}}\right),
$$

where $\Delta h_{f}$ is heat of formation, and $c_{p}$ is specific heat capacity at constant pressure for the premixed reactants, and $T_{\text {ref }}=0 \mathrm{~K}$.

Conservation of mass fraction of burnt fluid gives

$$
D f / D t=\dot{w},
$$

where $f=M_{\text {prod }} /\left(M_{\text {fuel }}+M_{\text {air }}+M_{\text {prod }}\right)$, and $\dot{w}$ is non-dimensional reaction rate. The kinematic boundary condition and no slip are given by, respectively,

$$
\boldsymbol{u} \cdot \boldsymbol{n}=\mathbf{0} \text { and } \boldsymbol{u} \cdot \boldsymbol{t}=\mathbf{0} \text { at all fixed walls. }
$$

The discrete approximation to the no-slip condition is satisfied within the numerical viscous sublayer, see $\S 5$. Consequently the no-slip condition is only posed on a finite part of the infinite channel with the cavity, see $\S 8$.

Uniform velocity at infinity is assumed:

$$
\boldsymbol{u}=U_{\infty} \boldsymbol{e}_{\boldsymbol{x}} \text { at } \pm \infty .
$$


Boundary conditions for scalar variables at all boundaries are

$$
\nabla F_{i}=0 \text { for } F_{i}=\rho, f, T, h,
$$

where $\rho$ is mixture density, $f$ is mass fraction of burnt fluid, $T$ is absolute temperature and $h$ is specific enthalpy.

The initial conditions are

$$
\begin{gathered}
u=U_{\text {pot }} \text { for } t=0 \text { (impulsive start), } \\
\rho=\rho_{\mathrm{u}}, \quad f=0, \quad T=T_{\mathrm{u}}, \quad h=c_{p} T_{\mathrm{u}} \text { for } t=0 .
\end{gathered}
$$

Ignition condition for the flow field:

$$
\rho=\rho_{\mathrm{b}} \text { for } t=\tau \text { in part of the fluid. }
$$

The volume expansion due to this initial burning is neglected.

\section{Numerical procedure}

According to Helmholtz's theorem the velocity field can be uniquely decomposed into a vorticity-free and a divergence-free component provided the divergence and vorticity approach zero at infinity. Consequently the two-dimensional velocity field can be written in the form:

$$
\boldsymbol{u}=\boldsymbol{\nabla} \varphi+\nabla \times \psi \boldsymbol{e}_{\boldsymbol{z}}
$$

It follows, taking the divergence and vorticity of this relation respectively, that

$$
\nabla^{2} \varphi=\nabla \cdot u \text { and } \nabla^{2} \psi=-\omega,
$$

where

$$
\omega=(\nabla \times u) \cdot e_{\boldsymbol{z}} .
$$

To satisfy the no-slip condition in the presence of rigid impermeable walls we must have

$$
\nabla \times \psi e_{z}=-\nabla \varphi \text { at the walls. }
$$

To satisfy the kinematic boundary condition conformal mapping theory was used. On the basis of (12)-(14) the known solution to an inviscid potential flow problem can be perturbed by adding vorticity and divergence into the solution of a simplified viscous flow problem where only one wall is present, and then transplanted onto the domain of the channel with a cavity to give the solution to this more complex viscous problem.

Using complex theory we replace (12) by

$$
u_{x}-\mathrm{i} u_{y}=\frac{d}{d \zeta}\left(W_{0}-\mathrm{i} W_{\omega}+W_{\varepsilon}\right) \frac{d \zeta}{d z},
$$

where $W_{0}, \mathrm{i} W_{\omega}$ and $W_{\varepsilon}$ are complex potentials giving the initial profile, the vorticity and the divergence perturbation respectively.

On the basis of (16) the problem is split into the following three sub-problems: (I) to determine uniquely the conformal mapping function $z=f(\zeta)$ and the initial velocity profile;(II) to determine the unsteady non-reacting flow; (III) to determine the unsteady reacting flow. These sub-problems are dealt with in $\$ \S 4-6$. 


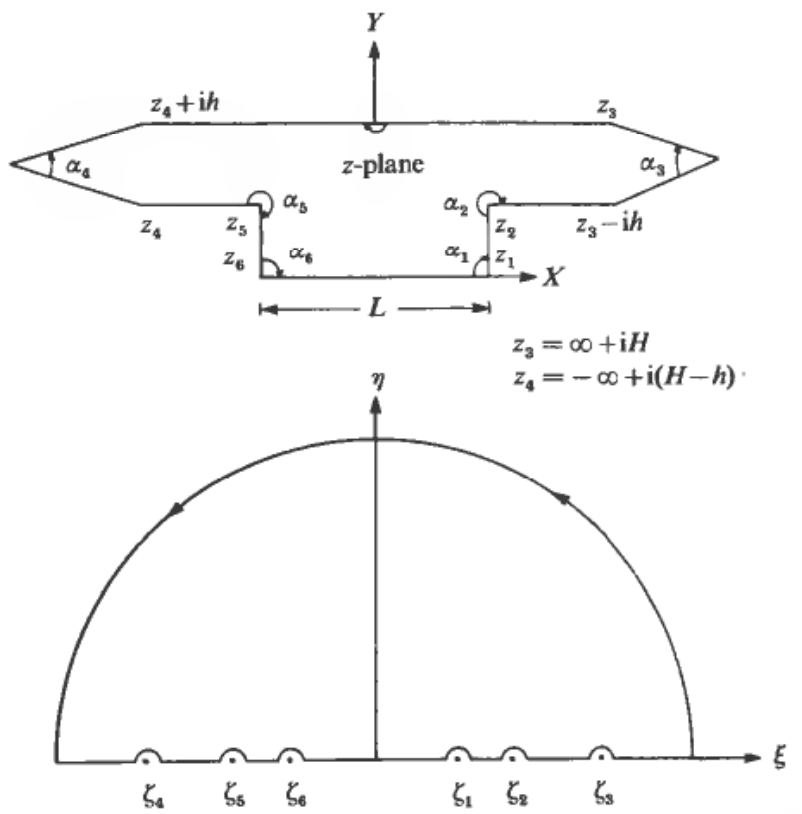

Figure 1. Computational domain in the $z$ - and the $\xi$-plane.

\section{Conformal mapping and initial profile}

The conformal mapping used is the Schwarz-Christoffel mapping function. The derivative of this mapping. Milne-Thompson (1968), can be expressed in the following form:

$$
\frac{d z}{d \zeta}=C \operatorname{cxp}(\mathrm{i} \lambda) \prod_{k=1}^{N}\left(\zeta-\zeta_{k}\right)^{\beta_{k}}
$$

where $\beta_{k}=\left(\alpha_{k} / \pi\right)-1, \alpha_{k}$ is the internal corner angle (in radians) for the corner number $k$, $C$ is a scaling parameter (real), $\lambda$ is a phase angle (real) and $\zeta_{k}$ is a point in the $\zeta$-plane on the closure of the domain $D$, corresponding to the corner $k$ of the domain $E$ in the z-plane.

Integrating (17) leads to the Schwarz-Christoffel mapping function:

$$
z=z_{0}+C \exp (\mathrm{i} \lambda) \int_{\zeta_{0}}^{\zeta}\left(\prod_{k=1}^{N}\left(\zeta^{\prime}-\zeta_{k}\right)^{\beta_{k}}\right) d \zeta^{\prime} .
$$

In order to apply (18) to our problem it is necessary to determine the parameters: $z_{0}, C, \lambda, \beta_{1}, \ldots, \beta_{N}, \zeta_{0}, \ldots, \zeta_{N}$.

The problem is overdetermined, so two points and the phase angle can be chosen at will. Our choice is $\lambda=\pi, z_{0}=0$ and $\zeta_{1}=1$. According to Riemann's mapping theorem, Henrici (1974), there will always exist a conformal analytic mapping which maps one to one the upper half-space $D(\eta>0)$ onto a polygon $E$ in the z-plane where the closure $E$ is not included. According to the Osgood-Caratheodory theorem, Henrici (1974), this transformation maps one to one the closure of the upper half-space onto the closure of the polygon chosen, provided the mapping is analytic in the upper unclosed half-space, and extends continuously to the closure. The proofs showing that the SchwarzChristoffel transformation satisfies these theorems for $D$ and $E$, both simply connected domains, are given in Henrici (1974). 
The integral (18) can generally not be inverted to find $\zeta=\zeta(z)$ for $N>2$. In our case $N=6$, see Fig. 1. Symmetry implies effectively $N=3$. Consequently an iteration procedure using geometrical information to check the deviation from the conformal mapping we want is needed, see Trefethen (1979).

To find the unknowns within a manageable number of iterations optimization theory is used. Our choice is to minimize the relative error in corner positions given by

$$
F\left(C, \zeta_{2}, \zeta_{3}\right)=\max \left[\left\|z_{1}-\frac{1}{2} L\right\|_{\mathrm{c}} / L_{\mathrm{ch}},\left\|z_{2}-\left(\frac{1}{2} L+\mathrm{i} H_{\mathrm{c}}\right)\right\|_{\mathrm{c}} / L_{\mathrm{ch}}\right],
$$

subject to the inequality constraints $\zeta_{3}>\zeta_{2}>1$. $L$ is the cavity length and $H_{\mathrm{c}}$ is the cavity height.

Using contour integration and the residue theorem it follows that $\zeta_{2}$ is related to $C$ and $\zeta_{3}$ through

$$
\zeta_{2}=\zeta_{3}\left[1-\left(\zeta_{3}^{2}-1\right) 4 H_{\mathrm{i}}^{2} /\left(\pi^{2} C^{2}\right)\right]^{1 / 2},
$$

where $H_{i}$ is inlet and outlet height.

It can be shown, Sand (1987), using the Kuhn-Tucker necessary conditions, Dorny (1975), that provided we stay within the feasible region, unconstrained optimization theory can be used.

We use a conjugate gradient descent method due to Fletcher and Reeves, see Dorny (1975), to determine a descent direction for $F$.

A modified version of Powell's line search, Dahlquist, Bjørk and Anderson (1974), is used for optimization of the descent step. Details of the computational procedure is given in Sand (1987).

The final transformation is then

$$
z=-\int_{0}^{\zeta}\left(C\left(\zeta^{\prime 2}-1\right)^{-1 / 2}\left(\zeta^{\prime 2}-\zeta_{2}^{2}\right)^{1 / 2}\left(\zeta^{\prime 2}-\zeta_{3}^{2}\right)^{-1}\right) d \zeta^{\prime},
$$

where $C=0.996697, \zeta_{2}=1.64281$ and $\zeta_{3}=1.93698$.

The maximum relative error in computed corner positions for the cavity using the procedure outlined is $1.2 \times 10^{-5}$.

Our polygon is closed at both $\pm \infty$, see Fig. 1. We therefore need a source and a sink with equal strengths to satisfy the continuity equation. Owing to our symmetry requirement we get the complex potential

$$
W_{0}(\zeta)=m \ln \left[\left(\zeta-\zeta_{3}\right) /\left(\zeta+\zeta_{3}\right)\right] \text {. }
$$

The dipole velocity field corresponding to this complex potential satisfies the kinematic boundary condition in the $\zeta$-plane except at $\pm \zeta_{3}$ which transform into $\pm \infty$ in the $z$-plane.

The outlet and the inlet boundary conditions $\nabla \varphi_{0}=U_{\infty} \cdot \boldsymbol{e}_{x}$ at $x= \pm \infty$ are satisfied provided the flux out of the source in the upper half- $\zeta$-plane is equal to the flux out of the channel in the $z$-plane. This determines the source strength $m=H_{\mathrm{i}} U_{\infty} / \pi$.

Using the conformal mapping (21), chain differentiation and the complex initial profile (22) we get

$$
u_{x 0}-\mathrm{i} u_{y 0}=2 H_{\mathrm{i}} U_{\infty}(\pi C)^{-1} \zeta_{3}\left(\zeta^{2}-\zeta_{2}^{2}\right)^{-1 / 2}\left(\zeta^{2}-1\right)^{1 / 2},
$$

where $C, \zeta_{2}$ and $\zeta_{3}$ are real variables. 


\section{Unsteady non-reacting flow}

\subsection{Velocity as a function of vorticity}

The velocity field is related to the vorticity distribution through a complex version of Biot-Savart's law:

$$
u_{x \omega}-\mathrm{i} u_{y \omega}=-\mathrm{i} \frac{d}{d \zeta}\left\{\int_{A} \int \frac{\Omega}{2 \pi} \ln \left[\left(\zeta-\zeta^{\prime}\right)\left(\zeta-\zeta^{\prime *}\right)^{-1}\right] d A^{\prime}\right\} \frac{d \zeta}{d z},
$$

where $\Omega$ is vorticity in the $\zeta$-plane and ${ }^{*}$ denotes complex conjugate.

The vorticity distribution in the $z$-plane is given by the non-dimensional Helmholtz vorticity equation for two-dimensional homogeneous incompressible flow:

$$
\frac{D \omega}{D t}=\frac{1}{R e_{\mathrm{ch}}} \nabla^{2} \omega .
$$

To solve this partial differential equation numerically without destroying the solution by numerical diffusion, we use the principle of fractional steps, Lie and Engel (1880). The splitting procedure applied (viscous splitting) consists of a convection fractional step followed by a diffusion fractional step.

This is written symbolically as

$$
\left\{\frac{\partial}{\partial t}-\frac{1}{R e_{\mathrm{ch}}} \nabla^{2}\right\}_{\mathrm{d}}\left\{\frac{D}{D t}\right\}_{\mathrm{d}} \omega=0
$$

where $d$ indicates that we consider a discrete form of the Helmholtz vorticity equation, and $D / D t$ means the substantial derivative.

A conceptual discussion of the procedure is given in the Appendix. Within the convection fractional step, vorticity is individually conserved; consequently the following Lagrangian formulation can be used for the convective vortex motion:

$$
\boldsymbol{r}_{i \mathrm{c}}=\int_{t_{i}}^{t} \boldsymbol{u}_{i} d t^{\prime}
$$

where $t_{i}$ is the time of birth for vortex $i$. To improve convergence of the method close to the boundaries the computational domain is split into an inner and an outer domain. The inner domain represents a numerical viscous sublayer next to all walls.

\subsection{Outer domain}

It can be shown using the Cauchy-Riemann conditions and Stokes' theorem that the circulation $\Gamma=\Omega d A$ is invariant with respect to the conformal mapping. Using a rectangular rule approximation and Chorin's cutoff for the singular vortex core we get

$$
\begin{aligned}
u_{x}-\mathrm{i} u_{y}=\left[\frac{d W_{0}}{d \zeta}-\mathrm{i} \sum_{j=1}^{N V(t)} \frac{\Gamma_{j}}{2 \pi}\left(\frac{\left(\zeta-\zeta_{j}\right)^{*}}{\left\|\zeta-\zeta_{j}\right\|}\right.\right. & \frac{1}{\max \left\{\left\|\zeta-\zeta_{j}\right\|, r_{\mathrm{c} \zeta}\right\}} \\
& \left.\left.-\frac{\left(\zeta-\zeta_{j}^{*}\right)^{*}}{\left\|\zeta-\zeta_{j}^{*}\right\|} \frac{1}{\max \left\{\left\|\zeta-\zeta_{j}^{*}\right\|, r_{\mathrm{c} \zeta}\right\}}\right)\right] \frac{d \zeta}{d z},
\end{aligned}
$$

where

$$
r_{\mathrm{c} \zeta}=r_{\mathrm{c}}\left\|\left.\frac{d \zeta}{d z}\right|_{\zeta_{j}}\right\|
$$

is used as the definition for the core radius in the $\zeta$-plane. 
It follows from the invariance of the complex potential under the transformation and the fact that a vortex does not induce velocity on its own centre, see Routh's rule, Milne-Thompson (1968), that the velocity of a vortex blob in the $z$-plane is given by

$$
u_{x k}\left(\zeta_{k}\right)-\mathrm{i} u_{y k}\left(\zeta_{k}\right)=u_{x}-\mathrm{i} u_{y}+\lim _{\zeta \rightarrow \zeta_{k}} \frac{\mathrm{i} \Gamma}{4 \pi} \frac{d}{d \zeta}\left\{\frac{d z}{d \zeta}\right\}\left(\frac{d \zeta}{d z}\right)^{2} .
$$

For Chorin's cutoff the last term is zero within the order of approximation, see Sand (1987).

The Simpsons fourth-order rule in time and a fourth-order Runge-Kutta in space give the following scheme for the vortex motion:

$$
z_{i}^{n+1}=z_{i}^{n}+\frac{1}{6}\left[u_{x_{i}}^{n}\left(\zeta_{i}^{n}\right)+4 u_{x_{i}}^{n}\left(\zeta_{i t t}^{n}\right)+u_{x_{i}}^{n}\left(\zeta_{i T p}^{n}\right)+\mathrm{i}\left\{u_{y_{i}}^{n}\left(\zeta_{i}^{n}\right)+4 u_{y_{i}}^{n}\left(\zeta_{i t t}^{n}\right)+u_{y_{i}}^{n}\left(\zeta_{i T p}^{n}\right)\right\}\right] \Delta t .
$$

(Since $u_{x}$ and $u_{y}$ are functions of $\zeta$ this scheme is equivalent to a fourth-order Runge-Kutta scheme.)

Here

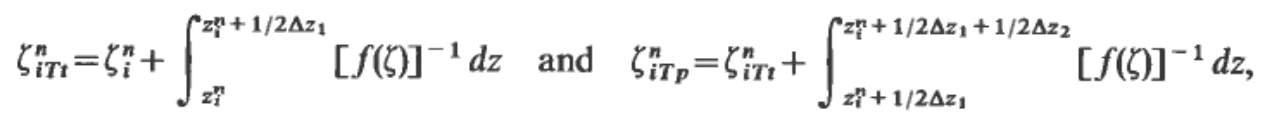

where

$$
\Delta z_{1}=\left[u_{x}\left(\zeta_{i}^{n}\right)+\mathrm{i} u_{y}\left(\zeta_{i}^{n}\right)\right] \Delta t \text { and } \Delta z_{2}=\left[u_{x}\left(\zeta_{i T t}^{n}\right)+\mathrm{i} u_{y}\left(\zeta_{i t t}^{n}\right)\right] \Delta t,
$$

$f(\zeta) \equiv d z / d \zeta$ is given by the derivative of the transformation.

The integrals are computed by using a fourth-order Runge-Kutta scheme. As a rule a minimum of 10 subintegration steps were taken during the computation.

The diffusion equation has a solution in the presence of boundaries given by generalized functions:

$$
\omega_{d}=\int_{A} \int \omega_{c}\left(A^{\prime}, t_{0}\right) G_{1}\left(A, A^{\prime}, t\right) d A^{\prime}+\int_{S} \int_{t_{0}}^{t} \frac{\partial \omega}{\partial t}\left(S^{\prime}, t^{\prime}\right) G_{2}\left(S, S^{\prime}, t^{\prime}\right) d t^{\prime} d S^{\prime},
$$

where $S$ is the closure of the domain $A$. The first term is due to the initial distribution of vorticity, while the second term is due to vorticity creation or destruction at the boundaries. Here

$$
\begin{aligned}
& G_{1}=\left(4 \pi t R e_{\mathrm{ch}}^{-1}\right)^{-1} \exp \left[-\frac{\left\{\left(x-x^{\prime}\right)^{2}+\left(y-y^{\prime}\right)^{2}\right\}}{4 t R e_{\mathrm{ch}}^{-1}}\right], \\
& G_{2}=\left(4 \pi\left(t-t^{\prime}\right) R e_{\mathrm{ch}}^{-1}\right)^{-1} \exp \left[-\frac{\left(r-r_{s}\right)^{2}}{4\left(t-t^{\prime}\right) R e_{\mathrm{ch}}^{-1}}\right] .
\end{aligned}
$$

It can be shown, see Chorin and Marsden (1979) for the first term and Sand (1987) for the second term, that $\omega_{d}$ can be represented stochastically by a random walk drawn from a normal distribution with a probability density function given by

$$
f=\left(4 \pi \Delta t R e_{\mathrm{ch}}^{-1}\right)^{-1} \exp \left[-\left(\xi^{2}+\eta^{2}\right) /\left(4 \Delta t R e_{\mathrm{ch}}^{-1}\right)\right]
$$

(zero mean value, and variance $\sigma^{2}=2 \Delta t R e_{\mathrm{ch}}^{-1}$, where $\Delta t$ is the discrete time step used for vortex motion). 
The final position of an individual vortex is then due to convection and diffusion (the latter represented by the random walk procedure):

$$
\boldsymbol{r}_{i}=\boldsymbol{r}_{i \mathrm{c}}+\xi_{i} \boldsymbol{e}_{\mathrm{x}}+\eta_{i} \boldsymbol{e}_{\boldsymbol{y}}
$$

$\xi_{i}$ and $\eta_{i}$ are here generated on the basis of (33) using a Box-Muller transformation and a random generator, Dahlquist et al. (1974).

\subsection{Inner domain}

To improve convergence close to boundaries a numerical viscous sublayer with thickness $\delta$ is defined where Prandtl's boundary-layer assumption is satisfied $(\partial / \partial x \sim \delta$ and $\delta \ll 1)$.

From the vorticity definition and the matching condition for the velocity field we get

$$
u(x, y, t)=u_{0 u}-\int_{y}^{\delta} \omega\left(x, y^{\prime}, t\right) d y^{\prime}+O\left(\delta^{2}\right) .
$$

The continuity equation and the kinematic boundary condition give

$$
v(x, y, t)=-\frac{\partial}{\partial x} \int_{0}^{y} u\left(x, y^{\prime}, t\right) d y^{\prime}+O\left(\delta^{2}\right)
$$

The detailed discretization procedure of (35) and (36) is given in Chorin (1978). This method is known as the vortex sheet method, and is adopted in this work for the inner domain.

The explicit forward Euler difference scheme in time and the random walk for the diffusion fractional step in the $y$-direction give the following algorithm for motion of vortex sheets within the viscous sublayer:

$$
(\boldsymbol{r})_{i}^{n+1}=(\boldsymbol{r})_{i}^{n}+(\boldsymbol{u})_{i}^{n} \Delta t+\eta_{i} \boldsymbol{e}_{y},
$$

where we have dropped the local subscript for notational convenience.

\subsection{Matching of the inner and the outer solutions}

The matching procedure used follows Cheer (1979). It ensures that the velocity field and the vorticity distribution per unit area are continuous at the interface between the inner and the outer domain, i.e.

and

$$
u_{0 \mathrm{u}}=u_{i} \text { at } y_{i}=\delta
$$

$$
\Gamma_{j}=\gamma_{j} h \text {. }
$$

Here $\Gamma_{j}$ is the circulation of vortex $j$ and $\gamma_{j}$ is the jump in velocity across the corresponding vortex sheet. It follows that the vortex core is given by

$$
r_{\mathrm{c}}=h / \pi \text {. }
$$

\section{Unsteady reacting flow}

\subsection{Velocity as a function of divergence}

The divergence distribution gives the following contribution to the velocity field:

$$
u_{x \varepsilon}-\mathrm{i} u_{y \varepsilon}=\frac{d}{d \zeta}\left\{\int_{V} \frac{E}{2 \pi} \ln \left[\frac{\left(\zeta-\zeta^{\prime}\right)\left(\zeta-\zeta^{\prime *}\right)}{\zeta^{2}-\zeta_{3}^{2}}\right] d V^{\prime}\right\} \frac{d \zeta}{d z},
$$

where $E$ is divergence in the $\zeta$-plane and ${ }^{*}$ denotes complex conjugate. 

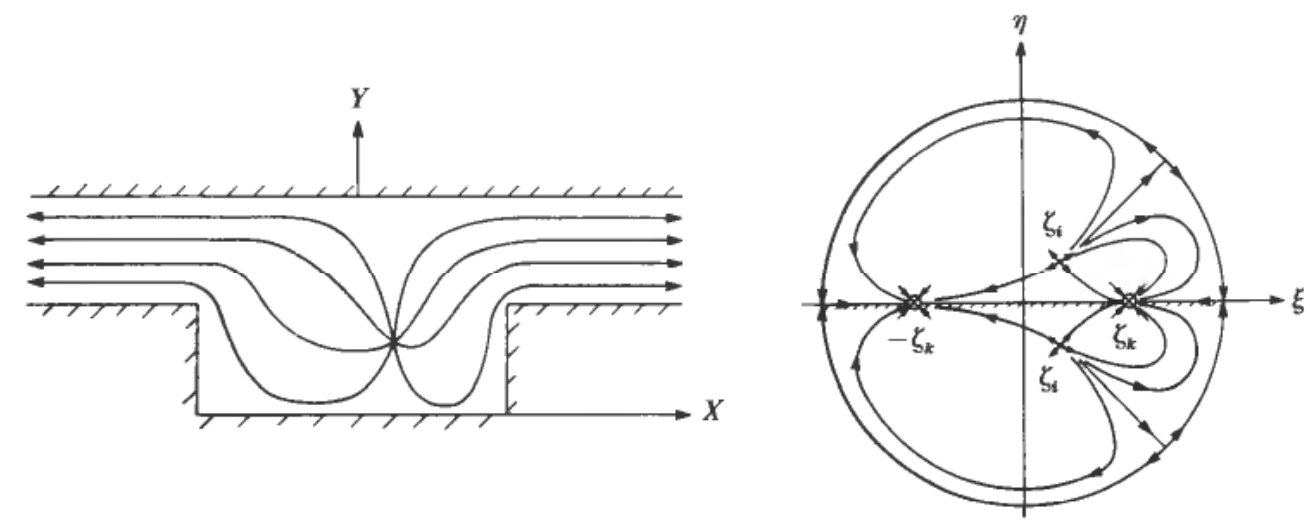

Figure 2. Particular solution of Poisson's equation for the volumetric expansion due to a combustion source in the channel with cavity.

It can be shown using the Cauchy-Riemann conditions and the Gauss theorem that the source strength $q=E d V^{\prime}$ is transformation invariant. Using a rectangular rule approximation and Chorin's cutoff for the core radius of the source we get

$$
\begin{aligned}
u_{x \varepsilon}-\mathrm{i} u_{y \varepsilon}=\sum_{k=1}^{N S(t)} \frac{q_{k}}{2 \pi}\left[\frac{\left(\zeta-\zeta_{k}\right)^{*}}{\left\|\zeta-\zeta_{k}\right\|}\right. & \frac{1}{\max \left\{\left\|\zeta-\zeta_{k}\right\|, r_{0 \zeta}\right\}} \\
& \left.-\frac{\left(\zeta-\zeta_{k}^{*}\right)^{*}}{\left\|\zeta-\zeta_{k}^{*}\right\|} \frac{1}{\max \left\{\left\|\zeta-\zeta_{k}^{*}\right\|, r_{0 \zeta}\right\}}-\frac{2 \zeta}{\left(\zeta^{2}-\zeta_{3}^{2}\right)}\right] \frac{d \zeta}{d z} .
\end{aligned}
$$

The last term ensures that the divergence goes to zero at infinity, see Fig. 2.

\subsection{Source strength}

The source strength and the core radius used are those of Ghoniem et al. (1982). They treat the flame as a one-dimensional deflagration wave. Details of their analysis are given in the same reference. The core radius is given by

$$
r_{0}=h_{\mathrm{cb}} / 2 \pi,
$$

where $h_{\mathrm{cb}}$ is the length and width of the square control volumes for motion of burnt fluid.

The source strength is given by

$$
q=\frac{1}{2} h_{\mathrm{cb}}^{2}\left\{\frac{\rho_{\mathrm{u}}}{\rho_{\mathrm{b}}}-1\right\} \frac{\Delta f}{\Delta t_{\mathrm{cb}}},
$$

where $f$ is the volume of burnt fluid within a combustion cell divided by the cell volume.

The ratio $\rho_{\mathrm{u}} / \rho_{\mathrm{b}}$ is in principle determined from (3) and (4) where $\Delta h_{\mathrm{f}}$ and $c_{p}$ are functions of the reactants used and the equivalence ratio chosen.

The rate of change in $f$ is determined within the computer code by the use of a simple line interface algorithm to represent the interface between burnt and unburnt fluid (the flame), and the use of Huygens' principle to move the flame normal to itself because of the local burning velocity.

The source strength used in this simulation stems from the one-dimensional analysis of Ghoniem et al. (1982). It is felt that it is appropriate to do an analysis based on mixture density and the continuity equation as well. 
We define the mixture density within a control volume $\Delta V$ as

$$
\rho=f \rho_{\mathrm{b}}+(1-f) \rho_{\mathrm{u}^{*}}
$$

From Leibnitz' rule, the Gauss theorem and the continuity equation in a coordinate system following the fluid we get

$$
\int_{V(t)} \frac{\partial \rho}{\partial t} d V=-\int_{S(t)}\left(\rho n \cdot u_{\mathrm{s}}\right) d S
$$

where $\boldsymbol{u}_{\mathrm{s}}$ is the relative velocity between the boundary and the fluid in the moving coordinate system. Assuming that the control volume used for combustion follows the fluid motion within the combustion fractional step we get

$$
q=\frac{h_{\mathrm{cb}}^{2}\left(\rho_{\mathrm{u}} / \rho_{\mathrm{b}}-1\right)}{f+(1-f) \rho_{\mathrm{u}} / \rho_{\mathrm{b}}} \frac{\Delta f}{\Delta t_{\mathrm{cb}}} .
$$

This source strength $q$ depends explicitly on $f$, unlike that of Ghoniem et al. We define

$$
k=q \Delta t_{\mathrm{cb}} / \Delta f
$$

The average value of $k$ with respect to $f \in[0,1]$ is then

$$
\bar{k}=h_{\mathrm{cb}}^{2} \ln \left(\rho_{\mathrm{u}} / \rho_{\mathrm{b}}\right)
$$

For $\rho_{\mathrm{u}} / \rho_{\mathrm{b}}=6, k=1 \cdot 8 h_{\mathrm{cb}}^{2}$. Therefore our $q$, which corresponds to $k=2 \cdot 5 h_{\mathrm{cb}}^{2}$ is $39 \%$ too high on the average. In the experiments of Vaneveld et al. (1982), flashback occurred for $\rho_{\mathrm{u}} / \rho_{\mathrm{b}}$ in the range of 7 to 7.5 depending on whether the walls were cooled or not (the latter case corresponds to adiabatic walls which is assumed in this work). The lower density ratio used partly compensates for the higher $q$ and reduces the deviation to $25 \%$.

\subsection{Flame motion}

In the thin reaction sheet regime for moderate turbulence intensity, the main effect of turbulence on the combustion is to increase the overall combustion rate by enhancing the flame area through wrinkling.

In agreement with the assumption of the fluid consisting of only two incompressible media, burnt fluid, the source term in the conservation equation for mass fraction of burnt fluid will always be located at the interface between burnt and unburnt fluid, and so will the divergence term in the conservation equation for mixture mass. Consequently these equations can be replaced by an interface-following algorithm with a divergence distribution at the interface representing the volume expansion due to the chemical reaction zone.

The interface representing the flame front is given by

$$
\frac{D \boldsymbol{r}_{\mathrm{f}}}{D t}=\boldsymbol{u}+S_{\mathrm{u}} \boldsymbol{n}_{\mathrm{f}}
$$

where $\boldsymbol{r}_{\mathrm{f}}$ is the position vector of an infinitesimal element of the interface, $S_{\mathrm{u}}$ is the local burning speed of the flame relative to the fluid motion and $\boldsymbol{n}_{\mathrm{f}}$ is a unit vector normal to the interface. 
The differential equation (50) can in discrete form be replaced by two fractional step operators:

$$
\left\{\frac{\partial}{\partial t}+S_{\mathrm{u}} n_{\mathrm{f}} \cdot \nabla\right\}_{\mathrm{d}}\left\{\frac{\partial}{\partial t}+u \cdot \nabla\right\}_{\mathrm{d}}\left(r_{\mathrm{f}}-r\right)=0
$$

where $\boldsymbol{r}$ is fixed in space, while $\boldsymbol{r}_{\mathrm{f}}$ follows the flame.

The first fractional step operator moves the flame through advection, while the second moves the flame relative to the fluid because of burning. In the first fractional step the mass fraction of burnt fluid is considered to be conserved individually with respect to the fluid motion and in the second with respect to the motion of the reaction zone relative to the fluid due to burning. This means that in the second fractional step the source term in the conservation equation for mass fraction of burnt fuel, which is located at the reaction zone, is treated as an equivalent convective term relative to the fluid.

\subsection{Numerical implementation of the flame motion}

A control volume approach is used for the conservation equation for mass fraction of burnt fluid. The mass fraction of burnt fluid is represented at the centre of the control volume, while a staggered grid is used for the velocities, see Fig. 3.

Chorin's extension of the simple line interface calculation algorithm of Noh and Woodward (1976), is used to represent the flame as an interface between burnt and unburnt fluid at subgrid level within a control volume. This is done with horizontal and vertical lines.

Pattern recognition is used to determine the form of the interface on the basis of volume fractions of burnt fluid in the neighbouring control volumes.

The simple line interface calculation algorithm recognizes for principally different configurations: a vertical interface, a horizontal interface, a rectangular corner and a neck, see Chorin (1980).

The neck is located by a constrained random choice, principally a sampling of Glimm's solution to a two-dimensional Riemann problem for a weak deflagration

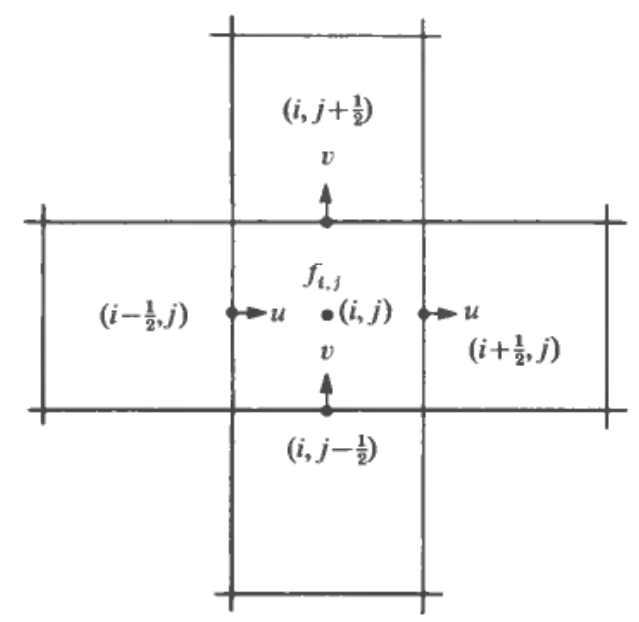

Figure 3. Grid showing where the velocities and the volume sources are computed during the flame motion. 
wave. The detailed requirements which the volume fraction of burnt fluid in the four neighbouring control volumes have to satisfy to recognize the listed configurations are given in Chorin (1980).

In the second fractional step the flame moves normal to itself owing to burning. Generally it is a difficult task to compute the normal unit vector to the interface which has to be simply connected. To avoid this difficulty Huygens' principle is used to locate the interface. The flame is moved in all directions (here eight are necessary to affect all neighbouring cells), and then the optimum spreading of the flame due to burning is chosen as the envelope condition.

To avoid directional preference a random angle $\in\left[0, \frac{1}{4} \pi\right]$ is added to each of the original eight directions within each combustion sub time step.

\section{Corner treatment}

In a potential flow problem a discontinuity in the boundary slope implies that the velocity in the fluid close to the discontinuity goes to infinity or to zero as we approach the singularity from the fluid side. Both cases are difficult to handle. We therefore want to get rid of these singularities locally. However, they are needed elsewhere in the flow to generate the right velocity field. Therefore we use locally a rounding technique given in Henrici (1974).

We distinguish between two cases, convex and concave corners seen from the fluid. The singular factor in the transformation is replaced by a polygon given by

$$
\left(\zeta-\zeta_{k}\right)^{\beta_{k}}=A(\zeta)\left(\zeta-\zeta_{k}^{\prime}\right)^{\beta_{k}}+B(\zeta)\left(\zeta-\zeta_{k}^{\prime \prime}\right)^{\beta_{k}}
$$

The concave corner seen from the fluid is given by (52) for $\beta_{k}>0$ and the convex corner for $\beta_{k}<0$. In order to remove the singularity from each of the terms we define

$$
A(\zeta)= \begin{cases}\left(\zeta_{k}^{\prime \prime}-\zeta_{k}\right)^{\beta_{k}}\left(\zeta-\zeta_{k}^{\prime}\right) /\left(\zeta_{k}^{\prime \prime}-\zeta_{k}^{\prime}\right)^{\beta_{k}+1} & \text { for } \beta_{k}<0 \\ \text { Constant } & \text { for } \quad \beta_{k}>0\end{cases}
$$

and

$$
B(\zeta)= \begin{cases}\left(\zeta_{k}^{\prime}-\zeta_{k}\right)^{\beta_{k}}\left(\zeta-\zeta_{k}^{\prime}\right) /\left(\zeta_{k}^{\prime}-\zeta_{k}^{\prime \prime}\right)^{\beta_{k}+1} & \text { for } \beta_{k}<0 \\ \text { Constant } & \text { for } \quad \beta_{k}>0\end{cases}
$$

Using the condition that (52) should be satisfied for $\zeta$ equal to $\zeta_{k}^{\prime}$ and $\zeta_{k}^{\prime \prime}$, and requiring in addition that $\zeta_{k}^{\prime}$ and $\zeta_{k}^{\prime \prime}$ are symmetrically located with respect to $\zeta_{k}$, gives

$$
\left(\zeta-\zeta_{k}\right)^{\beta_{k}}= \begin{cases}{\left[\left\{\frac{1}{2}\left(\zeta-\zeta_{k}^{\prime}\right)\right\}^{\beta_{k}+1}-\left\{\frac{1}{2}\left(\zeta-\zeta_{k}^{\prime \prime}\right)\right\}^{\beta_{k}+1}\right] /\left\{\frac{1}{2}\left(\zeta_{k}^{\prime \prime}-\zeta_{k}^{\prime}\right)\right\}} & \text { for } \quad \beta_{k}>0 \\ \left\{\frac{1}{2}\left(\zeta-\zeta_{k}^{\prime \prime}\right)\right\}^{\beta_{k}}-\left\{\frac{1}{2}\left(\zeta-\zeta_{k}^{\prime}\right)\right\}^{\beta_{k}} & \text { for } \quad \beta_{k}<0\end{cases}
$$

To avoid a priori separation of the boundary layer, the numerical viscous sublayer is defined as being connected past all internal corners of the flow. If a vortex sheet moves, in the local $x$-direction, a discrete convective fractional step sufficiently far to pass the corner and end in the outer flow region due to the corner geometry, it is placed in the local coordinate system past the corner as if the two local coordinate systems were connected without a corner in between. If however the vortex sheet has moved sufficiently far normal to the numerical viscous sublayer in the discrete time step for it to be located outside the numerical viscous sublayer, its global position will be relative to the corner, and become redefined as a vortex blob and its mirror image with respect to the axis $\zeta=0$. 


\section{Numerical simulation}

The numerical simulations consist of one simulation of unsteady flow of homogeneous incompressible fluid in a channel with a cavity, and one simulation of unsteady reacting flow in the same channel with a cavity, see $\S 9$.

\subsection{Characteristic scales and geometry}

The characteristic length $L_{\mathrm{ch}}$, velocity $U_{\mathrm{ch}}$ and time $T_{\mathrm{ch}}$ for the problem were chosen as maximum channel width $H$, initial inlet velocity $U_{\infty}$ and $H / U_{\infty}$, respectively. The channel with a cavity is symmetrical with respect to the $y$-axis with its inlet to the left, see Fig. 1. The dimensions of the part of the channel with the cavity considered are: Length $/ L_{\mathrm{ch}}=3.00$, width at cavity $/ L_{\mathrm{ch}}=1.00$, inlet height $/ L_{\mathrm{ch}}=0.50$ and cavity length $/ L_{\mathrm{ch}}=1 \cdot 42$.
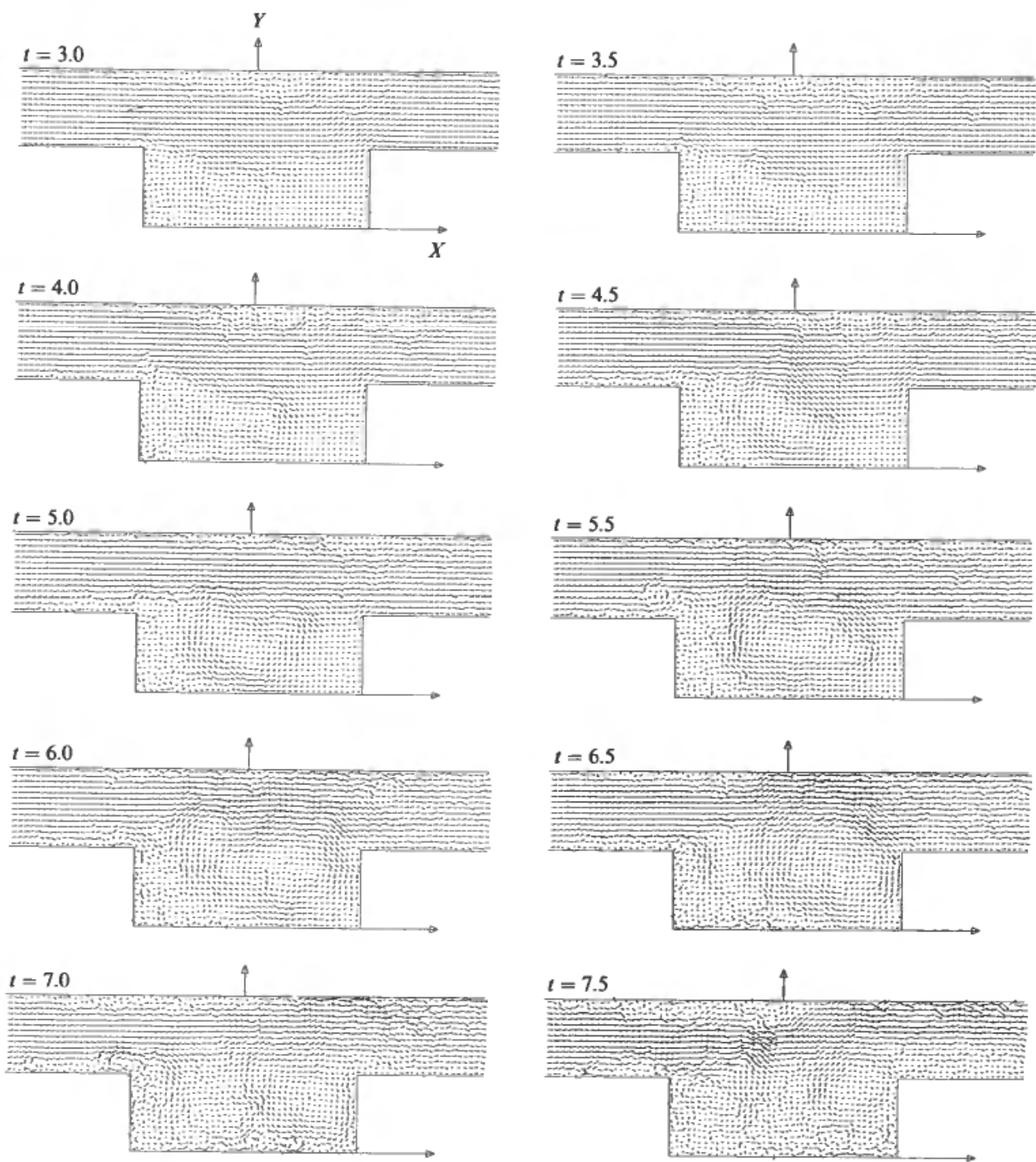

Figure 4. Time-development of the unsteady velocity field for non-reacting flow, at $R e=20000$,

$$
U_{\mathrm{ch}}=0.025 L_{\mathrm{cil}}
$$



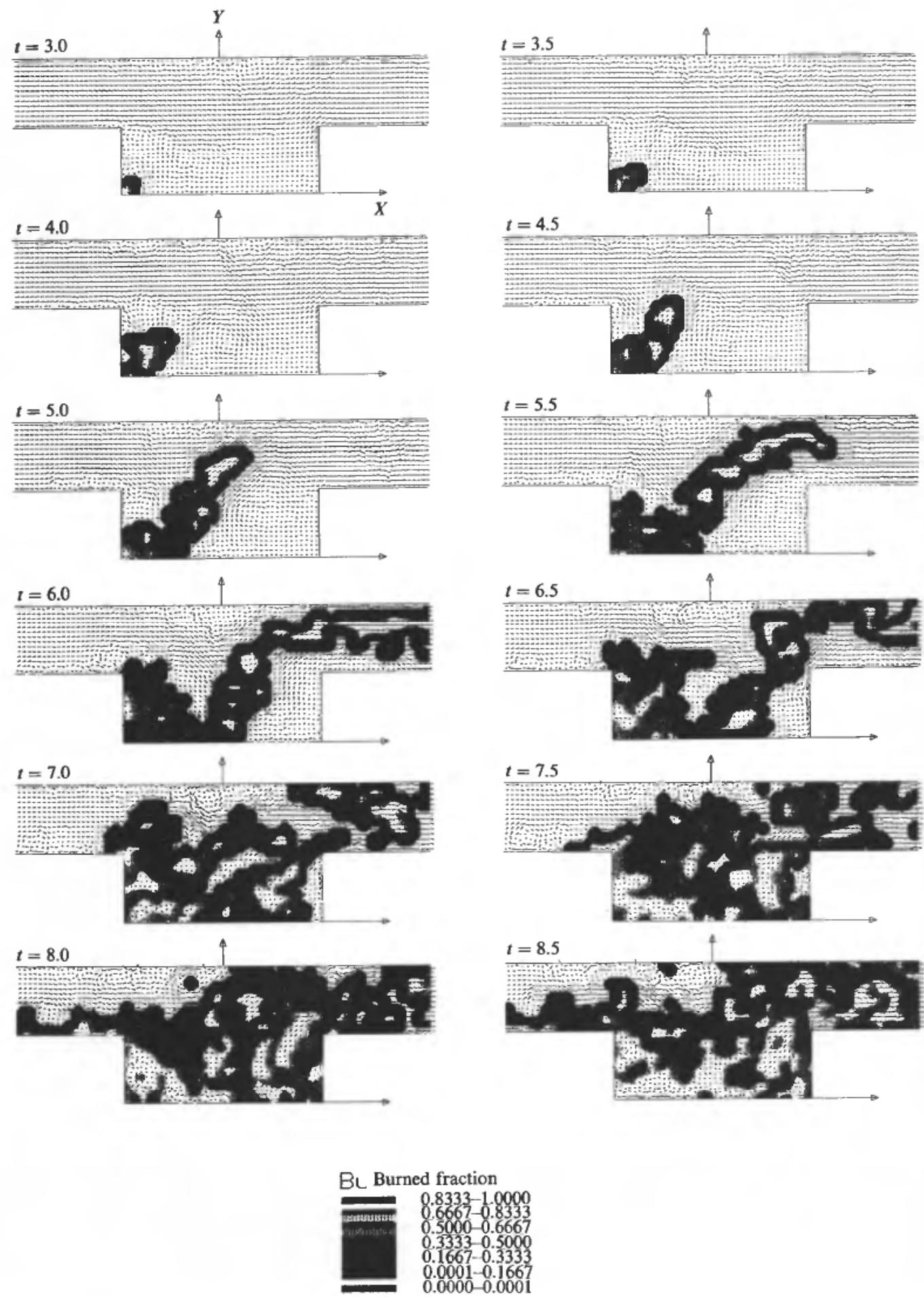

Figure 5. Time-development of the unsteady velocity field for reacting flow, and filled contour plots of the volume fraction of burnt fluid, at $R e=20000, U_{\mathrm{ch}}=0.025 L_{\mathrm{ch}}$. 

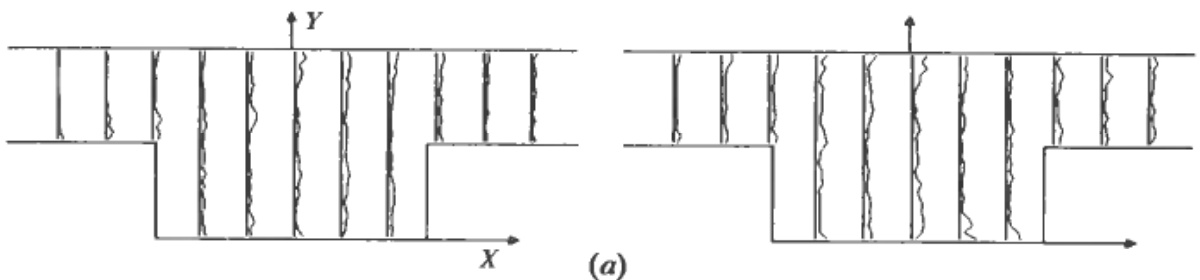

(a)
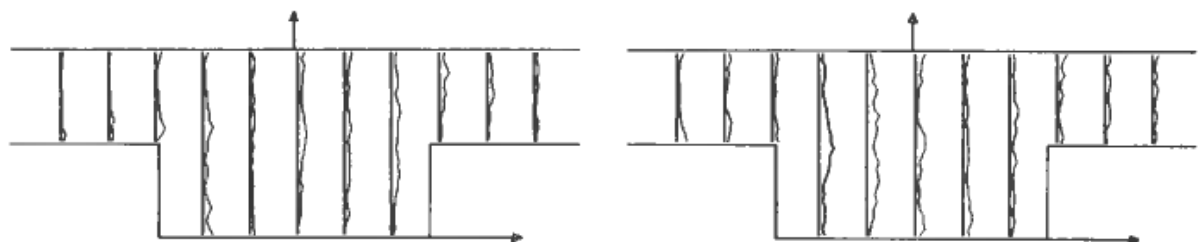

(b)

Figure 6. Time-development of the root-mean-square value of $(a)$ the $x$-component and $(b)$ the $y$-component of $\boldsymbol{u}^{\prime}$ for non-reacting flow, at $R e=20000, \Delta x / L_{\mathrm{ch}}=0.25$ and $U_{\mathrm{ch}}=0 \cdot 1 L_{\mathrm{ch}}$. Each curve represents the average of four samples taken at equally spaced points within the interval of time 4.5 to 6.0 (left-hand figures) and 6.0 to 7.5 (right-hand figures).
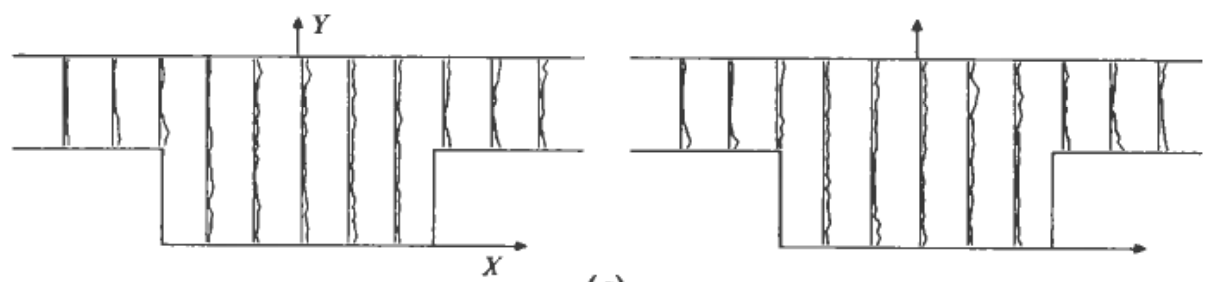

(a)
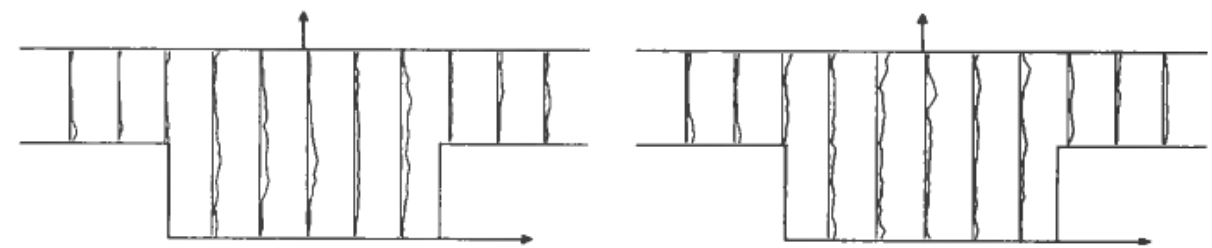

(b)

Figure 7. Time-development of the root-mean-square value of $(a)$ the $x$-component and $(b)$ the $y$-component of $u^{\prime}$ for reacting flow, at $R e=20000, \Delta x / L_{\mathrm{ch}}=0.25$ and $U_{\mathrm{ch}}=0-1 L_{\mathrm{ch}}$. Each curve represents the average of four samples taken at equally spaced points within the interval of time 4.5 to 6.0 (left-hand figures) and 6.0 to 7.5 (right-hand figures).

\subsection{Parameters used}

The non-dimensional time step for the vortex motion was chosen as $\Delta t=0 \cdot 1$. The non-dimensional thickness of the numerical viscous sublayer for all walls was chosen as $\delta=2 \sigma$, where $\sigma^{2}=2 \Delta t\left(R e_{\mathrm{ch}}\right)^{-1}$ is the variance of the diffusion fractional step of the vortex motion and $R e_{\mathrm{ch}}=U_{\mathrm{ch}} L_{\mathrm{ch}} / v$ is the characteristic Reynolds number. On the basis of the actual Reynolds number $U_{\mathrm{ch}} L_{\mathrm{ch}} /(2 v)=20000$ it follows that $\delta=2\left(5 \times 10^{-1}\right)^{1 / 2}$.

The non-dimensional vortex sheet length was chosen as $0 \cdot 1$, and the maximum and minimum sheet strengths per vortex sheet length were chosen to be 0.5 and 0.025 , respectively. The ratio between the time step for the deflagration wave relative to the fluid and the time step for the vortex motion was selceted as $\Delta t_{\mathrm{cb}} / \Delta t=0-2$. The gas is 


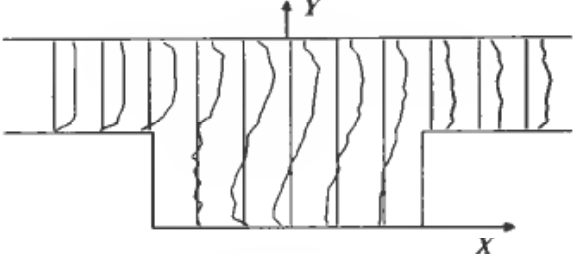

(a)

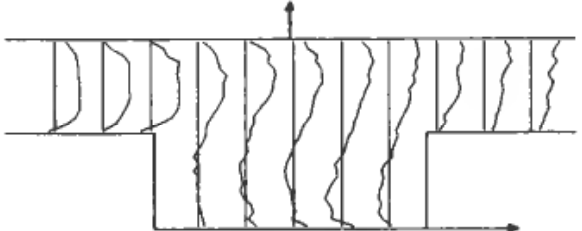

(b)

Figure 8. Time-development of the averaged streamwise velocity for non-reacting flow, at $R e=20000, \Delta x / L_{\mathrm{ch}}=0.25$ and $U_{\mathrm{ch}}=0 \cdot 1 L_{\mathrm{ch}}$. Each curve represents the average of four samples taken at equally spaced points within the interval of time (a) 4.5 to 6.0 and (b) $6 \cdot 0$ to $7 \cdot 5$.

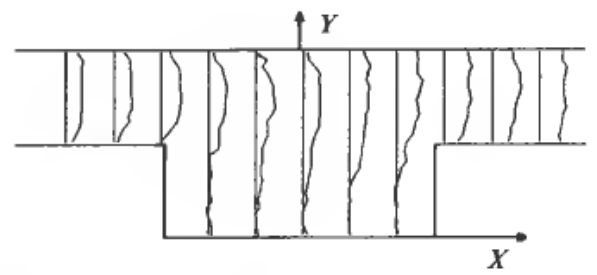

(a)

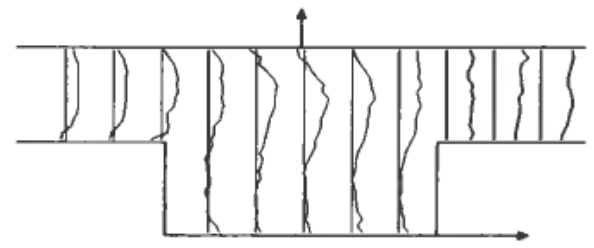

(b)

Figure 9. Time-development of the averaged streamwise velocity for reacting flow, at $R e=20000, \Delta x / L_{\mathrm{ch}}=0.25$ and $U_{\mathrm{ch}}=0.1 L_{\mathrm{ch}}$. Each curve represents the average of four samples taken at equally spaced points within the interval of time (a) 4.5 to 6.0 and (b) 6.0 to $7 \cdot 5$.

lean premixed propane and air with density ratio between unburnt and burnt fluid $\rho_{\mathrm{u}} / \rho_{\mathrm{b}}=6$ and corresponding non-dimensional local burning velocity $S_{\mathrm{u}}=0.02$. The non-dimensional length of the square combustion cell associated with a volume source was chosen as $h_{\mathrm{eb}}=0.0357$.

\subsection{Graphical presentation}

The results from the numerical simulations are presented as graphical plots. The velocity fields are visualized on a grid in the $z$-plane. The velocity field for non-reacting flow is given in Fig. 4. The scalar density field was represented by filled contour plots using a grey tone scale. This was done on the basis of the value of the volume fraction of burnt fluid within a combustion cell, represented as an average value at its centre, see Fig. 5. The root-mean-square values of $u^{\prime}$ and $v^{\prime}$ are shown for non-reacting and reacting flows in Figs 6 and 7, respectively. The averaged streamwise velocities for nonreacting and reacting flows are shown in Figs 8 and 9, respectively.

\section{Discussion}

\subsection{Unsteady flow}

A numerical simulation of a two-dimensional unsteady flow in a channel with a cavity has been carried out. The fluid is given an impulsive start as its initial condition. In principle the impulsively started flow problem has two solutions, one continuous and one discontinuous, where the streamlines bend and do not bend into the cavity, respectively. We assume that no corner is perfectly sharp and choose the continuous solution, see Fig. 10, as the initial condition. 


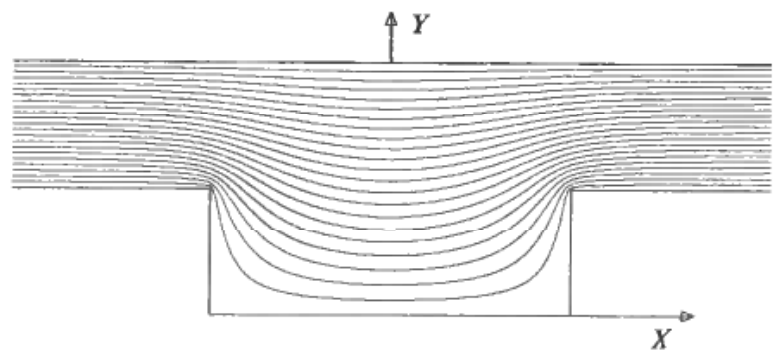

Figure 10. The continuous potential flow solution used as initial profile for the viscous flow problem.

When calculating unsteady flow downstream of a trailing edge the KuttaJoukowsky hypothesis can be used to show a boundary-layer spearation at the edge. The effect of viscosity on the outer flow field (the wake) can then be accounted for by generating discrete vortex blobs with cores sufficiently large to maintain a continuous smooth velocity at the separation point. However, in this problem where the position is not known a priori, or has an unsteady location, this strategy does not work.

The hybrid random vortex method can be used to solve this problem. Vortex sheets are generated to maintain no slip at locations distributed evenly along the wall. To avoid locating the separation point $a$ priori the vortex sheets can only leave the numerical viscous sublayer, which is defined also to exist past sharp corners, by motion normal to it.

When a vortex sheet leaves the numerical viscous sublayer it is redefined from only inducing velocity locally between itself and its normal projection on the wall to a vortex blob and its mirror image with respect to the wall location, which induce velocity globally. The vortex blob has a core with a size and strength designed to maintain the local tangential velocity smoothly after the redefinition, see $\$ 5 \cdot 4$. In consequence the inner solution, given by the vortex sheet distribution, combined with the matching procedure acts as a continuously distributed Kutta condition. Wherever a boundarylayer separation takes place, vortex sheets are converted into vortex blobs to account for the effect of viscosity on the flow field, which manifests itself as a wake downstream of the separation point.

Prandtl suggested zero shear stress at the wall as the criterion for separation of a boundary layer. It can be shown, Schlichting (1968), that separation of a boundary layer can only occur if the flow field is retarded. Through continuity, the widening of the channel retards the flow, while the narrowing downstream of the cavity accelerates it. Consequently, from a steady-state point of view, the boundary layer should separate at the upstream cavity edge and reattach at the downstream cavity edge. However, large vortex structures form and induce unsteady local retardation and acceleration as they move, so Prandtl's criterion for steady boundary-layer separation does not apply.

Moore, Rott and Sears, see Williams (1977), suggested a criterion which also covers unsteady separation. They assumed that separation would occur at a location of zero shear stress in a coordinate system moving with the speed of the separation point. This criterion reduces to Prandtl's separation condition in the steady situation. The simulated transition to fully developed unsteady flow is an impulsively started flow problem. Therefore some information can be extracted. The numerical simulation done for $R e=20000$ predicts separation of the boundary layer along the lower inlet wall at 
the step location, and for the boundary layer along the upper wall at the same $x$ location. This can be seen in the early stage of development of the flow field. Fig. 4, where plots of the velocity field show roughly the instantaneous locations of the separation points in the form of wakes.

In this early stage the boundary layer reattaches further downstream close to the cavity end. This is in agreement with our geometrical considerations, and Prandtl's criterion for a steady separated boundary layer. As the simulation evolves, the velocity field changes its character from steady to unsteady. From $t=4.5$ a large vortex structure is developing, see Fig. 4. The vortex structure accelerates the outer flow at the upper wall, and the boundary layer reattaches locally at approximately the same $x$ position as the centre of the vortex structure. From $t=6.5$ the large vortex structure approaches the downstream cavity corner. The vortex structure retards the flow at the lower outlet wall and accelerates the flow at the upper outlet wall. The boundary layer separates at the lower inlet wall and remains reattached at the upper wall.

The time-varying reattachment and separation are secondary effects induced by the vortex structures within the cavity. Since reattachment and separation points are moving as a consequence of the velocity induced by the large vortex structure, and seem to be roughly stationary seen from a coordinate system moving with the vortex structure, the simulation seems to be in agreement with the Moore, Rott and Sears' criterion for unsteady boundary-layer separation. However, this numerical simulation was not designed to study separation and reattachment of the boundary layer. Therefore quantitative results like zero shear at the edge of the visualized wake and the location of the saddlepoint for the streamlines at the separation point seen from the moving coordinate system, which are found in physical experiments, Ho (1986), are not computed or visualized, respectively.

Experimental results for comparison are not available for the channel with the cavity geometry. Pitz and Daily (1983) have done measurements of mean velocities and turbulence intensities in a combustion tunnel downstream of a rearward-facing step. The tunnel was three times deeper than it was wide, and therefore considered to be approximately two-dimensiona. One of their unsteady flow cases was carried out at $R e=22000$. Since Pitz and Daily did not have a cavity within their channel, only a sudden expansion of the channel, a comparison of location of reattachment points is irrelevant. However, quantitatively their average velocity profile, root-mean-square values of $u^{\prime}$ and turbulence intensities might have some similarity with ours. It should be noted that the narrowing of the channel by the cavity end constrains the width of the mixing layer downstream of the inlet cavity edge compared to the rearward-facing-step case. The trailing cavity edge also provides a possible feedback mechanism which does not exist with the rearward-facing step.

In Fig. 8 the average streamwise velocity for non-reacting flow is shown as a function of time. Only the last time interval, $t=6.0$ to 7.5 seems comparable with experiments, since the flow field within the other intervals is not fully developed. The recirculation behind the inlet cavity edge and behind the step are common for both geometries, see Pitz and Daily (1983). The simulated turbulence intensity in the transverse flow direction decays compared to that in the streamwise direction in the area close to the walls. This is also the case in Pitz and Daily's experiments. However, their turbulence intensities increase more distinctly towards the centre of the mixing layer. The failure to reproduce this tendency may be caused by the limitations imposed by the geometry on the length and width of the mixing layer, or by hydrodynamic interaction between the vortex structures inside the cavity and the boundary-layer 
separation upstream of the cavity forcing the separation to be unsteady. The nondimensional time step used in the simulation may also be too long to resolve the highest frequency content of the turbulence intensity, thereby limiting its size.

\subsection{Unsteady reacting flow}

The simulated unsteady two-dimensional flow field of a homogeneous incompressible fluid was used as the initial condition for the unsteady reacting flow problem. The fluid was assumed to consist of lean premixed propane and air. It was ignited at the lower inlet corner of the cavity at time $t=2.9$ by assuming four combustion cells filled with burnt fluid. The volume expansion due to the initial burning was neglected.

The unsteady non-reacting velocity field, used as the initial condition for the flame stability problem, was not fully developed. This can be seen from the turbulence intensity plots. The 'early' ignition was chosen to save computer time. It was assumed that the flame would not severely disturb the flow field during the first stage of development, $t=3.0$ to 5.0 . A comparison of the development of the simulated unsteady flow field with and without the combustion transient shows some difference in the recirculation pattern within the cavity. This is also indicated by the averaged velocities, see Figs 8 and 9 for averaged non-reacting and reacting flow, respectively.

From the simulations we see that the flame shoots up from the ignition corner of the cavity, basically rolling on a large recirculation vortex as it passes the upper corner of the outlet side of the cavity. The combustion rate then increases. The volumetric expansion, caused by the combustion, blocks off the flow field upstream and increases its speed downstream. Over time the flame crawls upstream along the separated boundary layer at the lower inlet wall. In effect the flame motion ends in a flashback.

Generally a flashback is considered to have four possible origins: auto-ignition upstream of the flame; classical flashback as a consequence of the local burning speed exceeding the flow velocity of the unburnt mixture either in the boundary layer or along the flow axis; flame propagation through reverse flow fields that may occur when the characteristic dimension $d$ of a disturbance is of the same order of magnitude as the distance $L$ between the disturbance and the flame holder, and pre-ignition of a separated flow region that may take place when $d$ is much less than $L$, Plee and Mellor (1978). In the present combustion model auto-ignition and pre-ignition are not taken into account. We are left with the classical flashback and disturbance resulting in reversed flow fields. Since we do not model acoustics, hydrodynamic oscillations are the only possible source for a disturbance. In fact hydrodynamic oscillations within the cavity interact with the boundary layer upstream, so a combined effect of the classical flashback and reversed flow due to hydrodynamic oscillations is possible.

The rolling of the flame on large vortex structures observed in the numerical simulation is in agreement with the experiments of Vaneveld et al. (1982). They use a Schlieren photographic technique to visualize the flame motion, and argue that the flame front basically stays parallel to the instantaneous streamlines of the vortex structures because of the small local burning speed. Therefore, the motion of the flame upstream of the inlet cavity edge can be considered to be a relay motion of the flame from one vortex structure big cnough to move the flame above the cavity edge, to a smaller vortex structure located in the unsteady separated boundary layer upstream of the cavity edge, which carries it further upstream. The simulation indicates that this is the case.

A second effect is the increased violence of the combustion due to vortices pulling out new branches of the flame surface leading to a dramatic increase of the flame area. 
Since the change in volume fraction of burnt fluid per unit time is proportional to the flame area, the volume expansion increases as well, and slows down the flow velocity in the upper part of the cavity, while increasing the flow velocity further downstream. The third effect is the unsteady boundary-layer separation, which makes it possible for the flame to separate the boundary layer further upstream as it moves within the wake of the boundary layer. The volumetric expansion retards the velocity field locally, as it moves with the flame, and thereby induces an unsteady separation of the boundary layer moving with the speed of the flame relative to a fixed reference frame. See the preceding section for a discussion of unsteady separation and reattachment.

However, this simulation can only be considered as an indication of the method's ability to simulate a flashback. It is possible that the simulated transient, though rather smooth due to the 'early' ignition and the drop of the initial volumetric expansion, has caused the necessary separation at the cavity edge. A complete simulation procedure to clarify this uncertainty would result from following the experiments of Vaneveld et al. (1982). Their combustion tunnel, which is three times deeper than it is wide, and therefore approximately two-dimensional, is in principle a channel with cavity. Their flame is first stabilized behind the upstream cavity edge and then triggered to flashback by a slight increase in the flow rate. Taking their channel width and inlet velocity our characteristic time $T_{\mathrm{ch}}=L_{\mathrm{ch}} / U_{\mathrm{ch}}$ is approximately $4 \mathrm{~ms}$. It follows that the part of the simulation which is of interest for the flashback lasts about $18 \mathrm{~ms}\left(T_{\mathrm{ch}}(8 \cdot 5-4 \cdot 0)\right)$. Experiments show that the interval of time which covers the transition to flashback is close to $280 \mathrm{~ms}$, see Schlieren photographs taken by Vaneveld et al. (1982). Of the hydrodynamically triggered phenomena reported by them, the 'chucking' has a time frame comparable to the flashback simulated.

To summarize: in this simulation we have used the combustion model of Ghoniem et al. (1982). To satisfy the kinematic boundary layer we have used a conformal mapping of the Schwarz-Christoffel type. In agreement with unsteady boundary-layer theory (Ho 1986, Williams 1977), we have avoided direct and indirect use of the KuttaJoukowski hypothesis (which breaks down in this case) by extending a numerical viscous sublayer continuously past the corners and also cutting off the corner singularities locally. In effect the boundary layer is free to separate anywhere. Therefore fluid dynamic oscillations within the cavity, manifested by vortex structures, are free to interact with the boundary layer and cause unsteady separation which is necessary to achieve a hydrodynamically triggered flashback as long as the net velocity upstream of the cavity inlet is higher than the burning velocity.

\section{Conclusions}

The numerical implementation of the hybrid random vortex method predicts, for a two-dimensional flow of homogeneous incompressible fluid, geometrically induced separation and reattachment of the boundary layer without the separation point being given a priori.

The numerical simulation resolves an unsteady flow field where vortex structures form and interact with the boundary layer and induce time-dependent separation and reattachment.

The main turbulence production within the flow field seems to be due to unsteady separation and unsteady reattachment of the boundary layer. The simulated turbulence intensities seem reasonable. However, the number of samples stored for analysis is too small to quantify the accuracy of the simulation performed. 
The low-Mach-number combustion model of Ghoniem et al. (1982) mimics the motion of a flame in a lean mixture of propane and air very nicely. In particular, the interaction between the flame and the boundary layer works well from a hydrodynamic point of view.

A complete simulation, where a flame is anchored downstream of the inlet cavity edge and then perturbed to flashback, has not been carried out. The simulation was disturbed by the combustion transient. However, a flashback of this type is a transient in itself, so the same mechanisms are present in both cases.

Hydrodynamic oscillations can lead to a flashback. However, the flashback seems to be due to a combination of three effects: a relay motion of the flame from one vortex structure to another, general blockage of the flow field caused by the volume expansion, and locally induced separation of the boundary layer ahead of the flame.

\section{ACKNOWLEDGMENTS}

This work has been financially supported by the Chr. Michelsen Institute, Department of Science and Technology, and by the Royal Norwegian Council for Scientific and Industrial Research. We have used a computer program due to Professor A. F. Ghoniem as a basis for the design of our computer program. This project is indebted to: Professor A. K. Oppenheim and Professor A. J. Chorin for discussions, encouragement and facilities provided at the University of California, Berkeley; Professor A. F. Ghoniem, MIT and Professor T. Ellingsen, University of Bergen, for encouragement and discussions; Professor B. H. Hjertager, Telemark Institute of Technology, Norway, and Dr R. K. Eckhoff at CMI Bergen, for supporting this project and for their help in initiating it.

\section{Appendix A. Comments on the numerical accuracy and convergence of the solution}

The convergence and accuracy of the simulated velocity field depend on the numerical scheme for the inner problem, the outer problem and on their matching procedure.

In the case of unsteady non-reacting flow, the numerical procedure simplifies to the hybrid random vortex method (Cheer 1979, Ghoniem et al. 1982). This method consists of the random vortex method (Chorin 1973) for the outer problem, and the vortex sheet method (Chorin 1978) for the inner problem.

The random vortex method and the vortex sheet method are in principle direct solution procedures for the Navier-Stokes equations through the Helmholtz vorticity equation and a relation between the vorticity distribution and the velocity field. The Helmholtz vorticity equation is solved in two fractional steps, a convective and a diffusive step. This 'viscous splitting' has the effect that the numerical diffusion within the convective terms does not compete with the diffusion terms. Owing to the splitting the numerical diffusion only alters 'the initial' conditions for the diffusion fractional step. In an unsplit solution procedure the diffusion term is $\propto 1 / R e_{\text {ch. }}$. For a large Reynolds number the numerical diffusion from the convective terms which is independent of the Reynolds number, will dominate the physical diffusion. In consequence 'The stochastic nature', of the deterministic solution, gets lost. The Lagrangian motion of individual vortices reduces in principle the numerical diffusion within the convective fractional step. However, if the numerical scheme used for the vortex motion is not accurate, an error acting as numerical diffusion will be introduced 
in the vortex distribution. To minimize this numerical diffusion the vortices were moved in the $z$-plane using a Simpsons fourth-order rule for the time integration. It is well known that in this case, where the velocity field is only a function of $\zeta$, see equation (31), this scheme is equivalent to a fourth-order Runge-Kutta scheme. Beale and Majda (1981) have shown that provided each of the fractional step problems converges globally, the random vortex method converges in time $\propto \Delta t / R e_{\text {ch. }}$. Hald (1979) has proved that the vortex method, with a given set of constraints on the vortex core, gives a velocity field which converges globally independent of time towards the solution of Euler's equations. The convergence in space is $\propto h^{2}$, where $h$ is the distance between the vortices. Hald (1985) later proved that the rate of convergence in the time variable is of fourth-order if the classical Runge-Kutta method is used, and the flow is sufficiently smooth. In particular Hald proves that the constraint he used on the vortex core in his earlier work, was too restrictive. His new proof also covers Chorin's 'cutoff' which is used in this work for the vortex core distribution. Majda and Beale (1982) have proved that a weight function can be used to construct vortex cores such that any space accuracy can be achieved.

The solution to the diffusion fractional step problem is approximated by the random walk, which is a stochastic representation of an analytic approximation to the solution. Chorin (1973) has estimated an error bound for the random walk as $\propto\left(1 / R e_{\mathrm{ch}}\right)^{1 / 2}$.

All the proofs and estimates are done without the presence of boundaries. The kinematic effect of a wall on the convection fractional step problem is in our simulation taken care of by including a mirror image distribution of the vortices with respect to the wall location wanted. A conformal mapping of the Schwarz-Christoffel type is used to transplant the velocity field induced by a vortex and its mirror image with respect to the wall onto the channel with cavity domain. This is in principle the same as adding an infinite series of vortex blobs and mirror images and their images etc. with respect to the wall locations. From a conceptual point of view this should not change the proved convergence of the velocity field derived from the convection fractional step vorticity distribution towards the solution of Euler's equations. Since the diffusion equation changes into another type of equation if the variables are transformed by a conformal mapping, a similar procedure cannot be used to handle the effect of the walls on the diffusion fractional step. However, locally in the physical plane, the wall effect is taken care of by a mirror image distribution whose effect is included by imposing reflection on the random walk. The matching zone at the edge of the inner and the outer flow domains acts as a sink and a source for vorticity for both fields, while the wall acts as a vorticity source for the inner field. Consequently the proofs and estimates referred to, which are all developed for an infinite space without walls and vorticity creation or destruction, do not in principle apply in our case. However, judging from the procedure used in these simulations to build up a unsteady flow, a sink for the vorticity field stabilizes the flow, while a source destabilizes it. The effect of the vorticity created at the wall is to perturb the potential flow through spreading of vorticity into an unsteady viscous flow. Therefore the creation of vorticity, which in principle is a destabilizing procedure, is correct as long as the perturbed potential flow converges towards the viscous unsteady flow as function of added vorticity. Chorin's vorticity creation procedure combined with the matching procedure for the inner and outer solution, acts in principle as a continuously distributed Kutta condition. If the flow field retards locally, a local separation of the boundary layer takes place because of the increased concentation of vortex sheets. The scparation is realized by a wake downstream of the 
separation point. This wake consists of vortex blobs, which have been converted from vortex sheets as they leave the numerical viscous sublayer. The vortex blobs induce velocity globally and retard the flow locally, maintaining the separation. If the flow is accelerated locally, reattachment of the boundary layer follows, realized by the disappearing of the wake. This is a consequence of a decreased concentration of vortex sheets locally. The mechanism necessary for keeping the vorticity perturbation of the potential flow under control such that the solution does not diverge, is therefore present.

The comments so far apply to the unsteady reacting flow field as well. However, the conservation equation for the volume fraction of burnt fluid is in principle solved by a control volume approach, and relies on a grid, unlike the random vortex method. Acoustic waves are not modelled, therefore numerical dissipation is synonymous with numerical diffusion. We can see the numerical diffusion manifest itself through a distributed reaction zone, see Fig. 5. However, part of this 'smearing' is caused by the contour plot routine used in the graphical presentation which does not recognize a sharp line interface as well as the simple line interface algorithm A smearing error is introduced by locating the velocity sources, representing the reacting flow, at the centre of the combustion cells. In agreement with the combustion model, which handles burnt and unburnt fluid outside the reaction zone as being incompressible, the volumetric sources should have been located at the interface, see Sethian (1984). An improvement can be achieved by locating each source through interpolation, at the averaged interface location within a cell containing part of the interface. The average combustion rate within the channel is possibly dependent on the grid resolution used for the Lagrangian motion of the flame. In principle, since the flame is assumed to be infinitesimally thin, there is no limit to how folded the flame area can become as function of the grid resolution used, and therefore there is no limit to how fast available fluid can burn. The principle limitation is therefore the entrainment of unburnt fluid through turbulent mixing. The turbulent mixing as represented here depends on both the grid resolution and the resolution of the turbulent velocity field. The time step for the vortex blob motion and circulation of the vortex blobs set an upper limit for the frequency resolution.

The numerical boundary conditions affect the accuracy of the solution. In this work the vorticity is set to zero downstream of the computational domain for unsteady viscous flow. This leads to inaccuracy in the resolution close to the downstream end of the domain. Our convenient artificial boundary condition can be replaced. Clements (1973) computed, in his vortex simulation of a free flow behind a thick splitter plate, the area-weighted average effect of clusters of vorticity. He followed them sufficiently far downstream to get negligible contribution from them in his defined area of interest. Basically this approach represents the same as continuing a rough Eulerian grid downstream for finite-difference schemes.

To maintain high accuracy in the motion of the discrete vortices, we have used Simpsons fourth-order rule for the time integration. It can be shown that, since the velocity field is a function only of $\zeta$, this scheme is equivalent to a fourth-order Runge-Kutta scheme. The use of a higher-order scheme is required to converge towards an unsteady flow field which is not particularly smooth, see Hald (1985). To maintain high accuracy in the correspondence between the $z$ - and the $\zeta$-plane we have used a fourthorder Runge-Kutta scheme with a non-dimensional space step equal to $\frac{1}{10}$ of the nondimensional time step. All numerical computations were carried out with doubleprecision accuracy. 
In Fig. 10 the streamlines for the continuous potential fiow solution are traced within the channel with a cavity, using in the conformal mapping a set of accessory parameters computed with the optimization procedure outlined briefly in $\S 4.1$. To check the accuracy of the transformation, the velocity due to the potential solution was computed at the walls. With the exception of the corner area and the area close to the point $x=0$ and $y=H$, the relative error is within $5 \times 10^{-2}$. The term 'error' here refers to the kinematic boundary condition which requires that the normal component of the velocity should be zero at the wall location.

The number of vortex blobs was $3000-4000$ for approximately fully developed flow, and the number of vortex sheets $1500-2500$. The number of burning combustion cells within each combustion time step $\Delta t_{\mathrm{cb}}$ was $400-600$ for the interval of time $t=7 \cdot 0$ to 8.5.

The flame was moved five sub steps $\left(\Delta t / \Delta t_{\mathrm{cb}}=5\right)$ between each change of the vortex field.

\section{REFERENCES}

ASHURST, W. T. and MCMURTRY, P. A. (1989). Flame generation of vorticity: vortex dipoles from monopoles. Combust. Sci Tech., 66, 17-37.

BEALE, J. T. and MAJDA, A. (1981). Rates of convergence for viscous splitting of the Navier-Stokes equations. Math. Comput., 37, 243-259.

BRAY, K. N. C. (1980). Turbulent flow with premixed reactants. In Turbulent Reacting Flows (ed. P. A. Libby and F. A. Williams). Topics in Applied Physics, vol. 44 (Springer).

CHEER, A. Y. (1979). A study of incompressible two-dimensional vortex flow past a circular cylinder. Lawrence Berkeley Lab. Rep., LBL-9950.

Chorin, A. J. (1973). Numerical study of slightly viscous flow. J. Fluid Mech., 57, 785-796.

Chorin, A. J. (1978). Vortex sheet approximation of boundary layers. J. Comput. Phys., 27, $428-442$.

ChORIN, A. J. (1980). Flame advection and propagation algorithms. J. Comput. Phys., 35, 1-11.

Chorin, A. J. and MardsDen, J. E. (1979). A Mathematical Introduction to Fluid Mechanics (Springer).

ClemenTS, R. (1973). An inviscid model of two-dimensional vortex shedding. J. Fluid Mech., 57, 321-336.

Dahlquist, G., BJork, $\AA$. and Anderson, N. (1974). Numerical Methods (Prentice-Hall).

DoRnY, C. N. (1975). A Vector Space Approach to Models and Optimization (Wiley-Interscience).

GANI, A. T. and SAWYER, R. F. (1980). Turbulence, combustion, pollutant and stability of a premixed step combuster. NASA Contractor Rep. 3230.

GHoniem, A. F., CHORIN, A. J. and Oppenheim, A. K. (1982). Numerical modelling of turbulent flow in a combustion tunnel. Phil. Trans. R. Soc. Lond. A, 304, 303-325.

HALD, O. H. (1979). Convergence of vortex methods for Euler's equations II. SI AM J. Numer. Anal., 16, 726-755.

HALD, O. H. (1985). Convergence of vortex methods for Euler's equations III. Rep. PAM-270. Center for Pure and Applied Mathematics (U. C. Berkeley).

HenRICI, P. (1974). Applied and Computational Complex Analysis, vol. 1 (John Wiley \& Sons).

Ho, C. M. (1986). An alternative look at the unsteady separation phenomenon. In Recent Advances in Aerodynamics (ed. A. Krothapalli) (Springer).

Keller, J. O., VANeveld, L., Korschelt, D., Ghoniem, A. F., Daily, J. W. and OPpenheim, A. K. (1981). Mechanism of instabilities in turbulent combustion leading to flashback. AIAA 19th Aerospace Sciences Meeting, AIAA-81-0107.

Law, C. K. (1988). Dynamics of stretched flames. 22nd Symp. (Intl) on Combustion, pp. 13811402. The Combustion Institute.

LIE, S. and ENGEL, F. (1880). Theorie der Transformation Gruppen (Leipzig: Teubner).

MAJDA, A. J. and BEALE, J. T. (1982). The design and numerical analysis of vortex methods. In Transonic, Shock and Multidimensional Flows (ed. Richard E. Meyer) (Academic).

Milne-Thompson, L. M. (1968). Theoretical Hydrodynamics, 5th edn. (Macmillan).

NoH, W. F. and Woodward, P. (1976). SLIC (Simple Line Interface Calculation). Proc. 5th Intl Conf. on Fluid Dynamics (ed. A. Vooren and P. Zandbergen), pp. 330-339 (Springer). 
PITZ, R. W. and DAlly, J. W. (1983). Experimental study of combustion in a turbulent free shear layer formed at a rearward-facing step. $A I A A J ., 21,1565-1570$.

PleE, S. L. and Mellor, A. M. (1978). Review of flashback reported in prevaporizing/premixing combustors. Combust. Flame, 32, 193-203.

SAND, I. Ø. (1987). On the unsteady reacting and non-reacting flow in a channel with cavity. Dr. Scient. thesis, University of Bergen, Norway.

Schlichting, H. (1968). Boundary-Layer Theory, 6th edn. (McGraw-Hill).

Sethian, J. (1984). Turbulent combustion in open and closed vessels. J. Comput. Phys., 54, 425-456.

Trefethen, L. N. (1979). Numerical computation of the Schwarz Christoffel transformation. ST AN-79-710.

VaneVeld, L., Hom, K. and Oppenheim, A. K. (1982). Secondary effects in combustion instabilities leading to flashback. AIAA 20th Aerospace Sciences Meeting. AIAA-82-0037.

Williams, J. C. (1977). Incompressible boundary-layer separation. Ann. Rev. Fluid Mech., 9, $113-144$. 